\title{
The Expression of VEGF and its Receptors in the Human Ductus Arteriosus
}

\author{
SVEN C. WEBER, CORNELIA RHEINLAENDER, NANETTE SARIOGLU, CHRISTIAN PEISER, MARIO RÜDIGER, \\ MICHAEL OBLADEN, AND PETRA S. KOEHNE
}

Departments of Neonatology [S.C.W., C.R., C.P., M.O., P.S.K.], Paidopathology and Placentology [N.S.], Humboldt University, 13353 Berlin, Germany; Department of Pediatrics IV and Neonatology [M.R.], Medical University Innsbruck, 6020 Innsbruck, Austria

\begin{abstract}
Programmed proliferative degeneration of the human fetal ductus arteriosus (DA) preceding definite postnatal closure has a large developmental variability and is controlled by several signaling pathways. Among these vascular endothelial growth factor (VEGF) and its receptors (VEGF-Rs) play an important role. Until now, gestational age dependent expression of VEGF and its receptors has not been investigated in a large number of human DA tissue specimens. We examined protein expression of VEGF and the three VEGF-Rs immunohistochemically in 63 human fetal autopsy DA specimens of 11-38 wk gestation. Specimens were classified into different maturity stages according to their histologic appearance. VEGF and VEGF-Rs-staining was detected in all maturity stages. VEGF-staining was localized perinuclearly in all vascular layers and did not change during development. VEGF-R1 and VEGF-R3 expression was marked in the endothelium in early maturity stages and decreased during development. In contrast, -R2 predominated in the media in later developmental stages. Our results emphasize the importance of VEGF as a mediator during programmed proliferative degeneration of fetal DA and support the hypothesis that VEGF-R1 and VEGF-R3 are required for normal blood vessel development during embryogenesis. In contrast, VEGF-R2 is the predominant receptor in later angiogenic signaling. (Pediatr Res 64: 340-345, 2008)
\end{abstract}

A abrupt increase in oxygen tension results in ductal constriction after birth. Falling levels of prostaglandin (PG) E2 due to enhanced metabolism in the lungs and elimination of the placental source contribute to an increase in ductal tone (1). In addition to PG, endothelin-1 seems to be involved in the primary functional closure of the DA $(2,3)$. DA medial smooth muscle contraction leads to wall thickening, luminal obliteration, and shortening of the vessel. The infolding of the endothelium, subintimal disruption and proliferation resulting in fibrosis lead to permanent sealing of the DA. The undisturbed interaction of these two processes, both functional and proliferative vascular obliteration, seems essential to the definite closure of the DA.

Failure of this complex process, initiated in part in utero, is associated with a patency of the DA after birth. A higher prevalence of patent DA is encountered in preterm infants

Received November 14, 2007; accepted April 29, 2008.

Correspondence: Petra Koehne, M.D., Department of Neonatology, Charité, Campus Virchow-Klinikum, Augustenburger Platz 1, D-13353, Berlin; e-mail: petra.koehne@ charite.de

Supported by grant from the Rahel-Hirsch research. The fluorescence microscope was purchased with a grant from the Sonnenfeld Stiftung (Berlin, Germany). than in term infants. Low oxygen tension and higher levels of circulating PG have been identified as major risk factors for insufficient ductal constriction in this population. The reasons for the failure of later definite obliteration of the DA in some preterm are not yet completely understood.

It seems that the hypoxia-inducible vascular endothelial growth factor (VEGF) functions as a critical regulator during programmed proliferative degeneration of the DA. VEGF regulates blood vessel development and homeostasis and is predominantly produced by endothelial and hematopoetic cells $(4,5)$. The biologic function of VEGF polypeptides is mediated by its binding to the type III receptor tyrosine kinases VEGF-R1 (Flt-1), VEGF-R2 (Flk-1/KDR), and VEGF-R3 (Flt-4) (6-9).

VEGF is found in the preterm and term DA of different mammalian species (10-12). Animal studies identified the formation of neointimal mounds and vasa vasorum ingrowth during permanent closure of the DA as VEGF-dependent processes (13). It is clear that VEGF expression in ductal smooth muscle cells is mediated by intense hypoxia within the constricted vessels. The time course of the expression of VEGF and its receptors in ductal tissue in late gestation is, however, poorly understood. It could be assumed that expression increases toward the end of pregnancy in preparation for spontaneous closure in term infants. Consequently, the failure of the duct to close permanently in some preterm infants could be due to low VEGF expression. The present study was performed to test the hypothesis that the expression of VEGF and its receptors in the endothelium and the media of the DA vessel wall varies between fetuses of a similar gestational and morphologic age. To answer this question, the maturitydependent protein expression of VEGF, VEGF-R1, VEGF-R2, and VEGF-R3 was studied in a large cohort of human fetal autopsy DA specimens with a gestational age (GA) ranging between 11 and $38 \mathrm{wk}$.

\section{METHODS}

Human tissue specimens. Autopsy specimens of the DA from 68 human fetuses and four lifeborns were collected over a period of $12 \mathrm{mo}$ at the Charité Department of Paidopathology. Informed parental consent was obtained before autopsy. The study was approved by the Charité Humboldt University ethics committee. Median GA was 22 wks (range, 11-38 wks). In addition to

Abbreviations: CHD, congenital heart defect; COX, Cyclooxygenase; PG, prostaglandin; VEGF-Rs, vascular endothelial growth factor receptors 
Table 1. Summary of clinical and pathological data from autopsy reports

\begin{tabular}{cccccr}
\hline Maturity score & Median GA (wk) & No. & Male/female & CHD & CMS \\
\hline I & 17 & 19 & $10 / 9$ & 5 & 8 \\
IIa & 22 & 29 & $21 / 8$ & 8 & 6 \\
IIb & 25 & 7 & $3 / 4$ & 2 & 11 \\
III & 35 & 8 & $4 / 4$ & 5 & 0 \\
\hline
\end{tabular}

Pathologic diagnoses summarized as CHD included Down syndrome with ventricular septal defect (two cases), hypoplastic left heart syndrome with (four cases) or without (one case) aortic valve stenosis or atresia, aortic valve stenosis (one case), coarctation of the aorta (two cases), pulmonary valve atresia (one case), pulmonary artery hypoplasia (one case), tetralogy of Fallot (two cases), double outlet right ventricle (one case), left ventricular rhabdomyoma (one case), and an acrofacial dysostosis with a complex vitium cordis including ventricular septal defect.

Pathologic diagnoses summarized as CMS included Potter-sequence (two cases), Down syndrome without cardiac malformation (two cases), neural tube defects (five cases), central nervous system malformations (7 cases), chromosomal aberrations without cardiac involvement (four cases), omphalocele/ gastroschisis (three cases), Pierre Robin sequence (one case) and skeletal malformations (three cases).

Cases not compatible with one of the two categories are summarized under "others." Pathological diagnoses in this group included placental insufficiency or placental ablation without infection (11 cases), cervical teratoma (one case), fetofetal transfusion syndrome (one case), nonspecified syndromes without heart defects (two cases), and four lifeborns with fatal outcome.

CHD, congenital heart defect; CMS, other congenital malformation syndrome.

medically indicated abortions, the main causes of death in the fetuses were amnion infection syndrome or other infections and placental insufficiency or placental ablation. Although spontaneous miscarriage predominated in the first trimester, intracervical application of synthetic PGE1 or PGE2 analogues was used to terminate pregnancies in the second trimester. In two thirds of the cases, either intrauterine death occurred or fetocide was performed before induction of labor by PG application. Among the four liveborns, one infant of 36-wk gestation died on d 3 of life from pulmonary artery embolism; another infant of 36-wk gestation died on d 9 of life in cardiorespiratory failure from sepsis; one infant born after 34 wks of gestation with gastroschisis died after 6 wks from necrotizing enterocolitis; and one infant with a GA of 38 wks died after $4 \mathrm{~h}$ due to a complex malformation with cardiac involvement.

Nine cases were excluded from VEGF and VEGF-Rs expression determination due to signs of inflammation and advanced autolytic degradation related to amnion infection syndrome. A frequency distribution of the clinical and pathologic diagnoses of the 63 cases that were included is summarized in Table 1. The fetuses were fixed in buffered $4 \%$ formaldehyde. After dissection and micropreparation under a stereomicroscope, the vascular DA tissue samples were embedded in paraffin, cut in 3-5 $\mu \mathrm{m}$ sections and mounted on coated slides (SuperFrost ${ }^{\circledR}$ plus TM, Langenbrinck Laborbedarf, Emmendingen, Germany).

Determination of ductal maturity. The Elastica van Gieson stained sections were evaluated as previously described (14) to assess histologic ductal maturity. Specimens were classified according to the histologic maturity score into one of the four maturity stages from early fetal life through to perinatal closure. Maturity-stage specific histologic characteristics of a stage I DA are: an endothelium in palisades, a well-defined media, absent intima cushions and an intact internal elastic lamina. In the DA maturity stage IIa, the endothelium becomes cubic oval and the internal elastic lamina shows interruptions. A flat endothelium, myocyte/fibroblast migration into the media and intima cushions locally reaching into the lumen are the characteristics of a IIb DA maturity stage. Fibromuscular remodeling of the media, extensive intima cushions and a fragmented internal elastic lamina characterize a III DA maturity stage. Histologic ductal maturity and immunofluorescence staining were assessed independently. Because of the significant morphologic differences between a DA just entering degenerative proliferation (stage IIa) and one in the middle of the process (stage IIb), the data from these two stages was analyzed separately.

Immunohistochemistry. Sections were deparaffinized in xylol twice for 10 min each, then rehydrated in ethyl alcohol $(100,90,80$, and 70\%), distilled water and PBS, for $3 \mathrm{~min}$ each at room temperature. VEGF, VEGF-R1, VEGF-R2, and VEGF-R3 staining was carried out after a standard protocol. For epitope retrieval, sections were fixed in citrate buffer $(\mathrm{pH} \mathrm{6.0)}$ at $600 \mathrm{~W}$ for $12 \mathrm{~min}$ in a microwave oven. The cooling sections were then washed three times in PBS. The specimens were incubated for $60 \mathrm{~min}$ in PBS $(0.1 \mathrm{M}, \mathrm{pH}$ 7.2) containing $2 \%$ goat serum, $1 \%$ BSA, $0.1 \%$ bovine gelatin, $0.1 \%$ Triton $\mathrm{X}-100$, and $0.05 \%$ Tween 20 with the objective of blocking unspecific proteins and increasing the permeability of the cell membrane, thus demasking intracellular epitopes. Sections were subsequently incubated overnight at $4^{\circ} \mathrm{C}$ with monoclonal mouse anti-human VEGF 1:500 (Santa Cruz Biotechnology Inc., Santa Cruz, USA), rabbit anti-human VEGF-R1, rabbit antihuman VEGF-R2, or rabbit anti-human VEGF-R3 primary antibody (Dianova, Hamburg, Germany) diluted in antibody diluent 1:100 (Zymed Laboratories, San Francisco, USA). For detection, a secondary fluorescent goat anti-mouse antibody or goat anti-rabbit (Alexa fluor ${ }^{\circledR} 488$ or 564 , MoBiTec GmbH, Goettingen, Germany) was applied at a dilution of 1:1000 in PBS and incubated at room temperature for $1 \mathrm{~h}$. After being washed three times with PBS, slides were counterstained and mounted with aqueous 4',6diamidino-2-phenylindole (DAPI) containing fluorescence protecting mounting media (Vectashield ${ }^{\circledR}$ hard set, Vector Laboratories, Burlingame, USA).

For double staining experiments, sections were blocked and primarily stained for VEGF before detection of VEGF-R1 or VEGF-R2 was performed. All steps were carried out as described above.

Human umbilical cord sections were used as positive controls in every experiment. The primary antibody was replaced by preimmune mouse or rabbit serum or by PBS alone for negative controls.

Morphometric analysis. VEGF, VEGF-R1, VEGF-R2, and VEGF-R3 staining intensity was evaluated by fluorescence microscopy. To this end, a representative section was photographed at two magnifications $(\times 200, \times 630)$ after immunohistochemistry using a fluorescence microscope (Microscope Axioplan 2e, Software Axiovision, Carl Zeiss, Jena, Germany). This guaranteed the consistent color intensity of the images and prevented a quality loss due to limited stability of the staining. The photographer (C.P.) was blinded to the phenotypical maturity of the specimens and did not participate in the evaluation. Immunofluorescence staining was evaluated semiquantitatively. On a discrete scale with 0.5 -intervals, points were given ranging from 0 for no staining to 3 for strong staining. The staining intensity was scored separately for two vessel regions: 1) the endothelium and subendothelial region and 2) the media. Histologic classification and immunofluorescence staining were independently rated by three blinded observers (C.R., S.C.W. and P.K.). Interobserver difference was 7.3\%. Differing results were discussed afterward until a consensus was reached. The Mann-Whitney-U-test was used for statistical evaluation, and $p<0.05$ was considered significant. Pearson correlation coefficients $\left(R^{2}\right)$ were calculated for associations between variables.

\section{RESULTS}

GA and histologic maturity. Figure 1 depicts GA frequency distribution as documented in the autopsy reports relative to

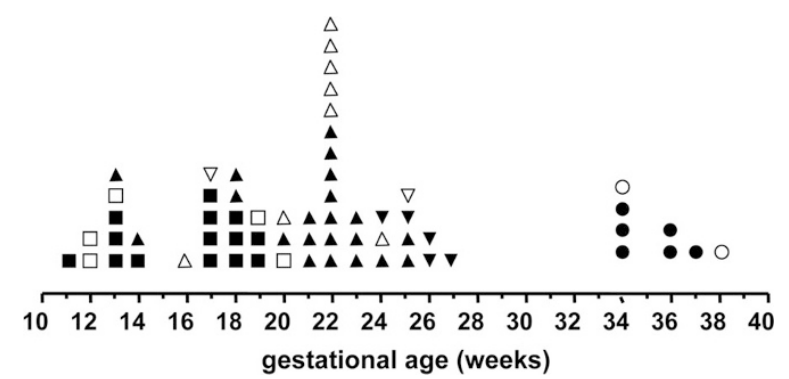

Figure 1. GA-dependent distribution of all cases relative to histologic maturity. Sixty three cases evaluated for protein expression of VEGF, VEGF-R1, VEGFR2, and VEGF-R3 are shown. Maturity stage I (squares) $n=19$, stage IIa (triangles pointing upward) $n=29$, stage IIb (triangles pointing downward) $n=$ 7 , stage III (circles) $n=8$. Empty symbols identify fetuses with CHD $(n=17)$, while filled symbols represent the diagnoses CMS and others $(n=46)$. 


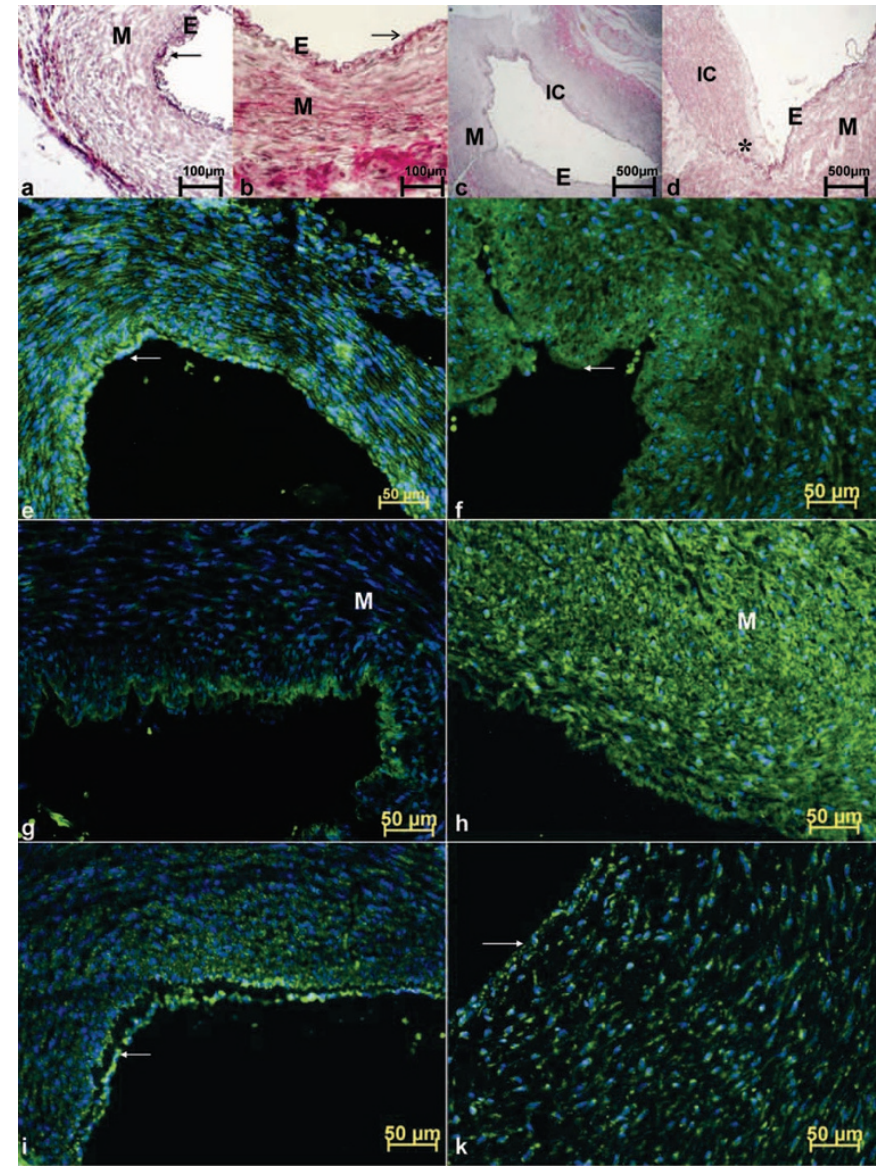

Figure 2. Staining of the DA in different maturity stages. Elastica van Gieson staining (upper row) depicts black elastic fibers, green nuclei, yellow erythrocytes, purple cytoplasm, and pink extracellular matrix. Light micrographs show different DA maturity stages I $(a)$, IIa $(b)$, IIb $(c)$ and III $(d)$. Maturity-stage specific histologic characteristics are present in endothelium ( $E$ or arrow), media $(M)$, intima cushions $(I C)$, and internal elastic lamina (*). The DA in maturity stage I still displays a regular organ architecture. Significant morphologic differences can be seen between a DA just entering proliferative degeneration (stage IIa) and one in the middle of the process (stages IIb and III). Immunofluorescence staining of VEGF-R1 (second row), VEGF-R2 (third row) and VEGF-R3 (lower row) shows green (Cy 2) positive cells with blue nuclei (DAPI-counterstained) in DA maturity stages I $(e, g, i)$ and III $(f, h, k)$. VEGF-R1 staining intensity was strongest in the endothelium of the early DA maturity stage (2e, arrow) and decreased significantly during development ( $2 f$, arrow). VEGF-R2 positive cells predominated in the DA media of the later maturity stage $(2 h, \mathrm{M})$. VEGF-R3 expression slightly decreased in the endothelium ( $2 k$, arrow) during maturation. The differences in staining intensity of the endothelium are marked with an arrow, whereas " $M$ " identifies the media of the DA.

the histologic maturity of each specimen. Two maxima occur around 18 and 22 wks of GA. According to our histologic maturity score, none of the DA specimens investigated complied with stage IV criteria (closed DA). The light micrographs in Figure. $2 a-d$ show the typical histologic features for each maturity stage. The GA and histologic maturity scores corresponded in $53(84 \%)$ of 63 cases $\left(R^{2}=0.85\right)$. Overall, seven specimens were judged histologically more mature and three less mature than the estimated date would indicate. The widest variance occurred between histologic maturity stages I and II. Six specimens were histologically classified stage II rather than stage I despite a lower GA. Data evaluation using

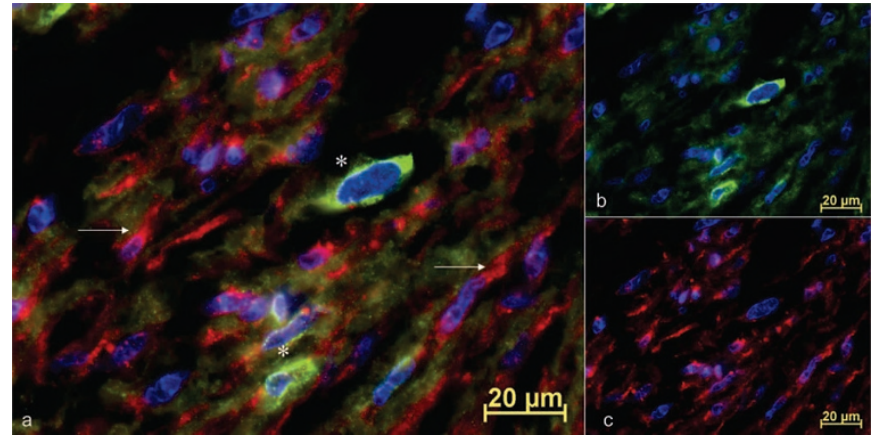

Figure 3. Double-immunofluorescence staining of a stage I DA. VEGF positive cells stain green (Alexa $488^{\circledR}$ ) and VEGF-R1 positive cells stain red (Alexa $564^{\circledR}$ ). Specimens are DAPI-counterstained (blue nuclei). The enlarged detail from the DA media region shows VEGF/VEGF-R1 double staining (a) with intracytoplasmatic VEGF-staining around the nuclei $(*)$ whereas VEGF-R1 is localized on the cell surface (arrow). The same localization pattern is seen in the micrographs of the single immunostaining for $\operatorname{VEGF}(b)$ and VEGF-R1 $(c)$.

either histologic maturity or GA resulted in corresponding results except for VEGF-R1 expression (see results paragraph on VEGF receptor-1 expression).

VEGF-expression. VEGF-positive cells were found in all maturity stages of the DA. VEGF-expression did not differ in the different maturity stages (data not shown). VEGF-positive cells were found in both the media and the endothelium of the vessels. There was no significant difference in staining intensity between the two regions. Intracellularly, VEGF-positive staining was primarily detected around the nucleus and did not change localization during development (Fig. $3 a$ and $3 b$ ).

VEGF receptor1-expression. The $180-\mathrm{kDa}$ glycoprotein VEGF-R1 was detected in all the maturity stages of the DA studied. The staining intensity was marked in the endothelium of the vessel wall in the early maturity stages. Endothelial VEGF-R1 expression decreased significantly during development and was minimal in stage III DA (Fig. 4 left column upper row, Fig. $2 e$ and $2 f$ ). We observed almost no change in the moderate expression of VEGF-R1 in the media throughout all maturity stages (Fig. 4 right column upper row, Fig. $2 e$ and $2 f$ ). A separate analysis of the six specimens judged more mature than GA alone would indicate (see Fig. 1 GA 11-19 wk) revealed a significantly lower expression of VEGF-R1 in the media of the DA than in the other 18 specimens with a GA ranging between 11 to 19 wks and DA stage I histologic maturity (median staining intensity 0.5 versus $1.0, p=0.018$ ). The same analysis showed no significant difference for the VEGF-R1 expression in the endothelium of the specimens with a GA of below 20 wks (median staining intensity 0.5 versus $1.0, p=0.077)$. At the cellular level VEGF-R1 was primarily expressed on the surface (Fig. $3 a$ and $3 c$ ).

VEGF receptor2-expression. VEGF-R2 positive cells were detected in both regions of the DA in all maturity stages (Fig. 4 lower row). Its expression in the endothelium did not change during DA development (Fig. 4, left column lower row). In contrast, the expression of VEGF-R2 significantly increased in the media in the later DA maturity stages and displayed the strongest staining intensity in maturity stages IIb and III (Fig. 4 , right column lower row, Fig. $2 g$ and $2 h$ ). Similarly to 

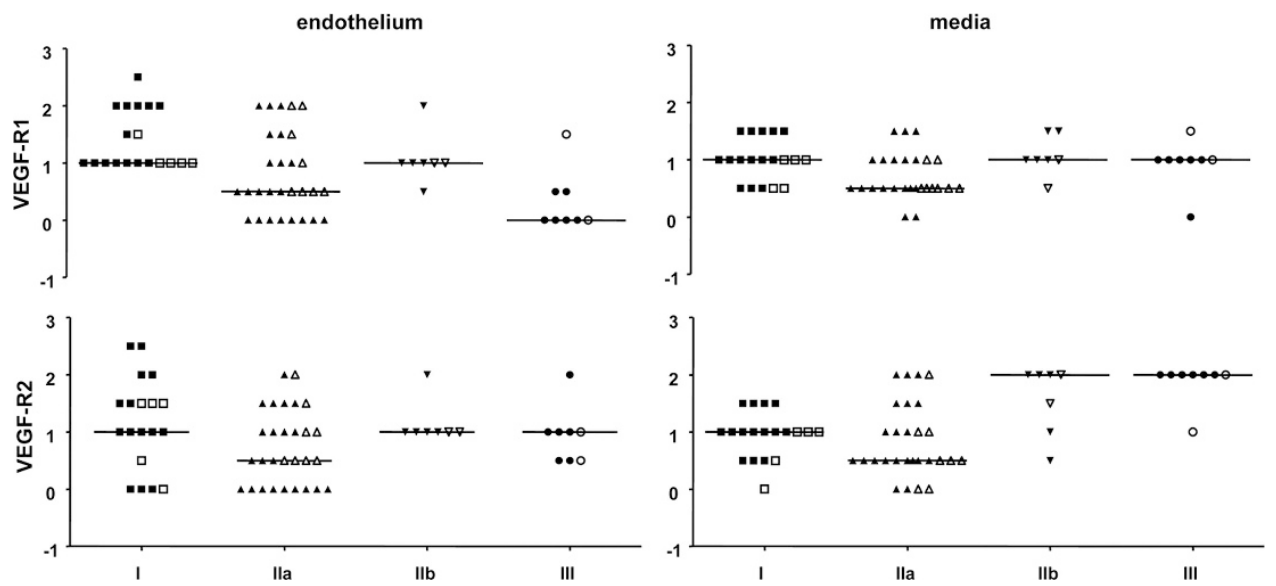

Figure 4. Maturity-dependent immunofluorescence staining intensity of VEGF-R1 and VEGF-R2 in human DA. Semiquantitative evaluation is expressed on a discrete scale ( $y$ axis) and separately scored for two vessel regions (left column, endothelium; right column, media). The $x$ axis depicts maturity stages. Maturity stage I (squares) $n=19$, stage IIa (triangles pointing upward) $n=29$, stage IIb (triangles pointing downward) $n=7$, stage III (circles) $n=8$, complete data shown as dot plots and median. Specimens of fetuses with CHD are indicated by the empty symbols in each of the four maturity stages (stage I $n=5$, stage IIa $n=8$, stage IIb $n=2$, stage III $n=2$ ). The expression of VEGF-R1 (upper row, left column) decreases significantly in the more mature stages in the endothelium (stage I vs. stage III, $p<0.05$ ). VEGF-R2-expression (lower row, right column) increases in the media of the vessel with higher maturity (stage I vs. stage III and stage IIa vs. stage III, $p<0.05)$.

VEGF-R1, the cellular localization of VEGF-R2 predominated on the cell surface (data not shown).

VEGF receptor3-expression. VEGF-R3 positive cells were detected in both regions of the DA in all maturity stages, although the staining intensity was stronger in the endothelium. During development a slight decrease of VEGF-R3 staining intensity was observed in the endothelium, especially when comparing maturity stages I and III (Fig. $2 i$ and $2 k$ ), though this was without statistical significance. Instead, the expression of the receptor in the DA media was consistently weak for all maturity stages.

Double staining experiments. Representative double staining experiments were carried out for VEGF and VEGF-R1 or VEGF-R2 to evaluate the co-expression of VEGF and its receptors along with their cellular distribution pattern in the DA. The cellular distribution pattern of VEGF, VEGF-R1, and VEGF-R2 remained unchanged throughout the different DA maturity stages. Although VEGF is primarily localized perinuclearly (Fig. $3 a, b$ ), VEGF-R1- (Fig. 3a, c) and VEGF-R2-expression are chiefly detected on the cell surface. The colocalization experiments for VEGF and VEGF-R1 or VEGF-R2 revealed a staining intensity and distribution pattern of the proteins in the two different DA vessel regions similar to the data analysis of the single staining experiments.

Specimens with CHD diagnosis. Congenital heart defects accounted for the major diagnosis in 17 (37\%) infants from our cohort of fetal autopsy specimens (empty symbols in Fig. 1). The broad spectrum of heart defects included in this group is listed in the legend of Table 1. A comparison of the expression of VEGF and VEGF-Rs in specimens with CHD to the other diagnosis groups revealed no differences for all proteins (empty symbols in Fig. 4 show expression of VEGF-R1 and VEGF-R2 in the four maturity stages for specimens with CHD). This also applies to the separate analysis of VEGF and VEGF-Rs expression in the DA for every maturity stage.

\section{DISCUSSION}

VEGF has been identified as a critical mediator for the remodeling of the DA that results in its permanent anatomical closure $(10,13,15)$. Programmed proliferative degeneration of the DA is a complex alteration process that begins during gestation. Because, VEGF plays a role in this process, a GA dependent change in the expression of VEGF and VEGF-Rs in the human DA vessel wall could be assumed to provide the basis for spontaneous ductal closure in term infants.

Interestingly, our study on human fetal DA autopsy specimens of various GA revealed homogenous VEGF expression in all DA tissue regions. This remained constant throughout all maturity stages. Although this result does not confirm our hypothesis, it conforms with the results of a few other studies that have detected VEGF in the muscle media of the preterm and term DA of various mammalian species (10-12).

Our study revealed a different expression pattern for all three VEGF-Rs in human fetal DA. Whereas VEGF-R1 expression was marked in the endothelium and subendothelial region of the immature DA and decreased during development (Fig. $2 e$ and $2 f$, Fig. 4), VEGF-R2 expression predominated in the media of more mature DA stages (Fig. $2 g$ and $2 h$, Fig. 4). In contrast, weak to moderate VEGF-R3 protein expression was observed. This decreased slightly in the endothelium in the advanced maturity stages and otherwise remained fairly constant (Fig. $2 i$ and $2 k$ ). A homozygous deletion of either VEGF-R1 or VEGF-R2 have been shown to be lethal in utero, since it results in severe malformations of the vasculature $(16,17)$. This would seem to indicate that both receptors are essential for the development of the vascular system during embryogenesis. This leads to the hypothesis that VEGF-R1 functions as a decoy receptor in early stages of embryogenesis to tightly control the concentration-dependent effect of VEGF (18). This might explain the higher expression of VEGF-R1 in the endothelium of our early gestation DA specimens. VEGFR-2, 
in contrast is the more "functional" receptor. It regulates endothelial cell migration, proliferation, differentiation and survival along with vessel permeability and dilation (6). These facts support our observation of predominant VEGFR-2 expression in the media of more mature DA specimens and correspond to the findings of Clyman et al. (13). They demonstrated a significant increase in vasa vasorum in the muscle media and an expansion of neointima thickness with smooth muscle cells following direct VEGF administration into the fetal lamb DA-wall. Although VEGFR-2 expression was not evaluated by Clyman et al., it seems likely that the observed structural changes were mediated by this receptor. Although immunohistochemical studies alone allow no conclusions to be drawn regarding the activity or functionality of a protein, the higher expression of VEGF-R2 in the media of our more mature DA specimens could reflect the first step in the anatomical DA remodeling process resulting in permanent closure. Our results, which demonstrate moderate and predominant VEGF-R3 expression in the early maturity DA endothelium that decreases during maturation, are in line with the known gestational changes in this receptor. It is initially expressed in all embryonic endothelial cells but is largely restricted to the lymphatic endothelium during later development (19).

Although the VEGF/VEGF-Rs system plays a key role in ductal closure, to date very little human data are available concerning the maturational changes in the expression of VEGF and its corresponding receptors. One likely reason for this is the difficulty in obtaining appropriate samples for investigation. The viable DA tissue that is required for molecular biology techniques is not excised during the surgical ligation of a persistent DA. This means that research on human DA tissue is limited to the immunohistochemical examination of postmortem specimens. Most specimens included in the present study were derived from medically indicated abortions with two maxima around 18 and 22 wks of GA. The gap in autopsy specimens between 28 and 33 wks reflects both the rarity of death and the difficulty in obtaining parental consent for a postmortem examination after a fatal clinical outcome of preterm infants in this GA range. These circumstances inevitably resulted in an uneven sample size in the different DA maturity stages, and may have influenced our results. With the exception of the four lifeborn infants included in the study, fetal specimens were investigated. This means that the present data on VEGF and VEGF-Rs expression is representative of intrauterine life. Questions regarding changes in the expression of VEGF and VEGF-Rs after exposure to higher oxygen concentrations that occur in postnatal life therefore remain unanswered.

In the present study, fetal autopsy specimens with documented vessel malformations at risk for higher circulating VEGF-levels (20) accounted for $37 \%$ of our cohort and were analyzed separately. A comparison of the expression of VEGF and VEGF-Rs in specimens with congenital heart defect to the other diagnosis groups revealed no differences during maturation for all proteins. This might in part be explained by the heterogeneity of our specimen group with regard to the inclusion of heart defects with or without involvement of the great arteries and ductus dependent circulation after birth. In contrast, the groups of Rabinovitch and
Gittenberger-de Groot found increased VEGF-expression to be related to the development of congenital heart defect in different animal models $(21,22)$.

Histologic maturity, which was determined independently of the protein expression of VEGF and its receptors, corresponded to GA in $84 \%$ of our specimens. The widest variance occurred between early histologic maturity stages where six specimens were histologically classified stage II rather than stage I and, despite their lower GA, had already entered the proliferative degradation phase characterized by morphologic changes beginning around 20 wks GA (Fig. $2 b$ ). This is in line with previous observations, where no strict correlation between GA and developmental maturity in human fetal and newborn DA tissue was observed (23). Nevertheless, we only found significant differences for the expression of VEGF-R1 between specimens in the same GA group below 20 wks. Expression of VEGF-R1 in the media of the DA was significantly lower in those specimens histologically judged more mature than indicated by GA. Otherwise, the present study shows a similar expression of VEGF and its receptors for similar GA and fails to explain the clinical observation of a different reaction of the DA in preterm infants despite the same GA. Our results emphasize the importance of the evaluation of histologic ductal maturity since structural changes explain different target protein expression in DA specimens of the same GA.

In conclusion, our study describes the developmental expression of VEGF and the VEGF-Rs in human DA autopsy specimens. We observed a spatio-temporal variation of VEGF-Rs expression rather than a continuous perinuclear VEGF expression in all DA layers throughout gestation. Although endothelial VEGF-R1 and VEGFR-3 expression declined with increasing GA, VEGF-R2 expression predominated in the media of more mature DA stages, indicating the later as the main receptor for VEGF-mediated structural DA changes.

Our study has a descriptive character. Further functional studies, e.g. animal models, are necessary to clarify the interaction between the differential expression of VEGF and its receptors. These would help determine whether the expressional changes also modulate the receptors affinity to VEGF binding as well as their implications for permanent postnatal closure of the DA.

Acknowledgments. We thank Evelyn Strauß for her assistance in preparing sections for immunohistochemistry from the paraffin embedded specimens. We thank Sarah SmithsonCompton for suggestions regarding the English.

\section{REFERENCES}

1. Schneider DJ, Moore JW 2006 Patent ductus arteriosus. Circulation 114:1873-1882

2. Taniguchi T, Azuma H, Okada Y, Naiki H, Hollenberg MD, Muramatsu I 2001 Endothelin-1-endothelin receptor type A mediates closure of rat ductus arteriosus at birth. J Physiol 537:579-585

3. Keller RL, Tacy TA, Fields S, Ofenstein JP, Aranda JV, Clyman RI 2005 Combined treatment with a nonselective nitric oxide synthase inhibitor (1-NMMA) and indomethacin increases ductus constriction in extremely premature newborns. Pediatr Res 58:1216-1221

4. Ferrara N, Davis-Smyth T 1997 The biology of vascular endothelial growth factor Endocr Rev 18:4-25

5. Leung DW, Cachianes G, Kuang WJ, Goeddel DV, Ferrara N 1989 Vascula endothelial growth factor is a secreted angiogenic mitogen. Science 246:1306-1309

6. Cebe-Suarez S, Zehnder-Fjallman A, Ballmer-Hofer K 2006 The role of VEGF receptors in angiogenesis; complex partnerships. Cell Mol Life Sci 63:601-615 
7. Shibuya M 2001 Structure and function of VEGF/VEGF-receptor system involved in angiogenesis. Cell Struct Funct 26:25-35

8. Kabrun N, Buhring HJ, Choi K, Ullrich A, Risau W, Keller G 1997 Flk-1 expression defines a population of early embryonic hematopoietic precursors. Development 124:2039-2048

9. Ishida A, Murray J, Saito Y, Kanthou C, Benzakour O, Shibuya M, Wijelath ES 2001 Expression of vascular endothelial growth factor receptors in smooth muscle cells. J Cell Physiol 188:359-368

10. Clyman RI, Chan CY, Mauray F, Chen YQ, Cox W, Seidner SR, Lord EM, Weiss H, Waleh N, Evans SM, Koch CJ 1999 Permanent anatomic closure of the ductus arteriosus in newborn baboons: the roles of postnatal constriction, hypoxia, and gestation. Pediatr Res 45:19-29

11. Kajino H, Chen YQ, Seidner SR, Waleh N, Mauray F, Roman C, Chemtob S, Koch CJ, Clyman RI 2001 Factors that increase the contractile tone of the ductus arteriosus also regulate its anatomic remodeling. Am J Physiol Regul Integr Comp Physiol 281:R291-R301

12. Seidner SR, Chen YQ, Oprysko PR, Mauray F, Tse MM, Lin E, Koch C, Clyman RI 2001 Combined prostaglandin and nitric oxide inhibition produces anatomic remodeling and closure of the ductus arteriosus in the premature newborn baboon. Pediatr Res 50:365-373

13. Clyman RI, Seidner SR, Kajino H, Roman C, Koch CJ, Ferrara N, Waleh N, Mauray F, Chen YQ, Perkett EA, Quinn T 2002 VEGF regulates remodeling during permanent anatomic closure of the ductus arteriosus. Am J Physiol Regul Integr Comp Physiol 282:R199-R206

14. Rheinlaender C, Weber SC, Sarioglu N, Strauss E, Obladen M, Koehne P 2006 Changing expression of cyclooxygenases and prostaglandin receptor EP4 during development of the human ductus arteriosus. Pediatr Res 60:270-275

15. Levin M, McCurnin D, Seidner SR, Yoder B, Waleh N, Goldbarg S, Roman C, Liu BM, Boren J, Clyman RI 2006 Postnatal constriction, ATP depletion, and cell death in the mature and immature ductus arteriosus. Am J Physiol Regul Integr Comp Physiol 290:R359-R364

16. Fong GH, Rossant J, Gertsenstein M, Breitman ML 1995 Role of the Flt-1 receptor tyrosine kinase in regulating the assembly of vascular endothelium. Nature 376:66-70

17. Sakurai Y, Ohgimoto K, Kataoka Y, Yoshida N, Shibuya M 2005 Essential role of Flk-1 (VEGF receptor 2) tyrosine residue 1173 in vasculogenesis in mice. Proc Natl Acad Sci USA 102:1076-1081

18. Dumont DJ, Fong GH, Puri MC, Gradwohl G, Alitalo K, Breitman ML 1995 Vascularization of the mouse embryo: a study of flk-1, tek, tie, and vascular endothelial growth factor expression during development. Dev Dyn 203:80-92

19. Kukk E, Lymboussaki A, Taira S, Kaipainen A, Jeltsch M, Joukov V, Alitalo K 1996 VEGF-C receptor binding and pattern of expression with VEGFR-3 suggests a role in lymphatic vascular development. Development 122:3829-3837

20. Ootaki Y, Yamaguchi M, Yoshimura N, Oka S, Yoshida M, Hasegawa T 2003 Vascular endothelial growth factor in children with congenital heart disease. Ann Thorac Surg 75:1523-1526

21. Rabinovitch M 2007 Pathobiology of pulmonary hypertension. Annu Rev Pathol 2:369-399

22. van den Akker NM, Molin DG, Peters PP, Maas S, Wisse LJ, van Brempt R, van Munsteren CJ, Bartelings MM, Poelmann RE, Carmeliet P, Gittenberger-de Groot AC 2007 Tetralogy of fallot and alterations in vascular endothelial growth factor-A signaling and notch signaling in mouse embryos solely expressing the VEGF120 isoform. Circ Res 100:842-849

23. Desligneres S, Larroche JC 1970 Ductus arteriosus. I. Anatomical and histological study of its development during the second half of gestation and its closure after birth. II. Histological study of a few cases of patent ductus arteriosus in infancy. Biol Neonate 16:278-296 